\title{
Consideraciones económicas sobre la salud pública cubana y su relación con la salud universal
}

\author{
Ana María Gálvez González, ${ }^{1}$ Roxana González López, ${ }^{2}$ Manuel Álvarez Muñiz, ${ }^{3}$ \\ María Josefina Vidal Ledo, ${ }^{1}$ Nery de la Concepción Suárez Lugo ${ }^{1}$ y Mayelín \\ Vázquez Santiesteban ${ }^{3}$
}

Forma de citar

Gálvez González AM, González López R, Álvarez Muñiz M, Vidal Ledo MJ, Suárez Lugo NC, Vázquez Santiesteban M. Consideraciones económicas sobre la salud pública cubana y su relación con la Salud Universal. Rev Panam Salud Publica. 2018;42:e28. https://doi.org/10.26633/RPSP.2018.28

RESUMEN Este trabajo expone, en el contexto de la estrategia de salud universal, algunas consideraciones generales y acciones económicas sobre el financiamiento, la eficiencia y otros desafíos económicos de la salud pública en Cuba. Se revisaron fuentes bibliográficas oficiales del estado cubano hasta 2015. Los análisis de series temporales se ajustaron a precios constantes de 1997 y tasa oficial de cambio: 1 peso cubano = 1 dólar estadounidense. El Sistema Nacional de Salud cubano cuenta con una infraestructura sólida, constituida por una amplia red de instalaciones sanitarias que garantizan la cobertura total, el acceso y la equidad en los servicios de la población con recursos humanos calificados. Se realizó un análisis económico bajo el marco conceptual de la estrategia de salud universal con énfasis en el financiamiento, la eficiencia y los desafíos de la salud pública cubana para mantener el derecho a la salud de sus ciudadanos, la equidad, cobertura y acceso en los servicios que se prestan, incluso la eliminación de las barreras económicas, socioculturales y de género, así como la solidaridad con la población cubana y del mundo. Se concluyó que en Cuba se considera la salud universal como una de sus más valiosas realizaciones, aunque se requiere del análisis más eficiente de fuentes fiables y disponibles, la investigación y aplicación de resultados a la práctica sobre las fortalezas y debilidades del sistema en materia de economía de la salud, actualización y uso de los recursos y tecnologías, así como profundizar en las lecciones aprendidas y enfrentar un conjunto de desafíos.

Palabras clave Financiamiento en salud; eficiencia; economía de la salud; Cuba.

Cuba participa de la Estrategia de Salud Universal que promueven la Organización Mundial de la Salud (OMS) y la Organización Panamericana de la Salud (OPS) (1, 2). Su Sistema Nacional de Salud (SNS) es integrado, universal y gratuito, con tres niveles de atención. Para

\footnotetext{
Escuela Nacional de Salud Pública, Cuba.

2 Organización Panamericana de la Salud/Organización Mundial de la Salud, Cuba.

3 Área de Economía del Ministerio de Salud Pú-
} blica, Cuba. ello, cuenta con un área básica de 451 policlínicos, cuya célula principal son los consultorios que tienen al personal médico y de enfermería requerido en vinculación directa con la población, entre otras instituciones destinadas al primer nivel de atención. Además, dispone de 150 hospitales en el segundo nivel de atención y 19 instituciones de investigación y atención en el tercer nivel. Esto constituye una sólida infraestructura, con recursos humanos calificados ${ }^{4} \mathrm{y}$ articula una amplia red de instalaciones sanitarias que garantizan la cobertura y el acceso plenos para toda la población $(3,4)$.

\footnotetext{
4 Aproximadamente medio millón de trabajadores de la salud incluidos 90161 médicos, para una proporción de 80,2 médicos por 10000 habitantes, a los que corresponde en promedio la atención de 125 habitantes por médico (Anuario Estadístico de Salud de Cuba, 2017).
} 
Después de 1959, Cuba alcanzó indicadores sanitarios comparables con los de países de mayor desarrollo económico. Los nuevos grandes desafíos del siglo xxI para la salud pública cubana no radican en mejorar el acceso, la cobertura o los indicadores sanitarios más importantes, sino en encontrar reservas de eficiencia.

Bajo los efectos de la crisis económica internacional y del bloqueo comercial y económico por parte de los Estados Unidos de América, Cuba lleva a cabo un proceso de actualización de su modelo económico que, a pesar de exhibir logros en los ámbitos político y diplomático, no ha encontrado aún solución a los problemas de la dualidad monetaria y cambiaria, la baja productividad o la inversión extranjera insuficiente, entre otros. También afronta el envejecimiento de su población, la baja fecundidad y las dificultades inherentes a los procesos migratorios (3-5).

En la actualidad, el SNS en la actualidad está abocado a un claro llamado a la eficiencia sin menoscabo de la excelencia en los servicios. En respuesta a ello, a partir del 2011 se inició la implementación de un plan de perfeccionamiento y transformaciones dirigido a reorganizar, compactar y regionalizar los servicios de salud con el fin de "consolidar sus acciones e incrementar la efectividad y la eficiencia de los planes y programas, así como la capacidad del sistema para enfrentar retos previsibles relacionados con el envejecimiento poblacional y otras contingencias de salud, que podrían derivarse del cambio climático y sus consecuencias" (6).

El objetivo de este trabajo es presentar, en el contexto de la estrategia de Salud Universal, algunas consideraciones generales y acciones económicas sobre el financiamiento, la eficiencia y desafíos de la salud pública en Cuba.

\section{Desarrollo}

El artículo se basa en el resultado de un estudio de revisión de fuentes bibliográficas oficiales del Estado cubano hasta el 2015; se utilizan datos de la Oficina Nacional de Estadísticas e Información (ONEI), documentos del Ministerio de Salud Pública (MINSAP), de las agencias internacionales de cooperación, así como otras referencias internacionales sobre Cuba según las dimensiones del SNS en el contexto de la Salud Universal, financiamiento y eficiencia.
Para todos los análisis que lo requirieron se trabajó con la tasa oficial de cambio del peso cubano (CUP) a dólares estadounidenses (USD); a razón de 1:1. Los análisis de las series temporales se ajustaron a precios constantes de 1997; para ello, se emplearon los deflactores del producto interno bruto (PIB) utilizados por la ONEI $(7,8)$.

\section{Cuba en el contexto de la Salud Universal}

Los estados miembro de la OPS forman un conjunto heterogéneo que incluye naciones muy desarrolladas, como los Estados Unidos de América y Canadá, y países pobres como Haití; estados de gran extensión territorial y pequeñas islas en el Caribe. Sus pueblos hablan distintas lenguas y tienen una gran diversidad étnica y cultural; algunos conservan vivos sus orígenes indígenas y otros se han nutrido fundamentalmente de la inmigración, con una fuerte influencia del Viejo Mundo.

El derecho a la salud, la equidad y la solidaridad constituyen los pilares básicos de la Salud Universal. Estos tres valores aportan el fundamento ético de las políticas públicas en salud. El atributo "universal" incluye tanto la cobertura como el acceso y pone especial énfasis en la eliminación de las barreras económicas, socioculturales y de género que limitan dicho acceso. También incluye, entre sus líneas estratégicas, ampliar el acceso equitativo a servicios de salud, fortalecer la rectoría, la gobernanza y la coordinación intersectorial, aumentar el financiamiento y mejorar la eficiencia.

El financiamiento y la eficiencia constituyen el centro de atención de este artículo, que examina varios de sus elementos de consenso: eliminar el pago directo como barrera para el acceso, incrementar el gasto público en salud hasta $6 \%$ del PIB, usar financiamiento mancomunado solidario, aumentar la eficiencia del sistema de salud y racionalizar la incorporación de tecnologías (2).

Una mirada al financiamiento en salud en el contexto regional permite constatar diferencias entre los países, en relación con las fuentes de los recursos. En algunos países, como Colombia y Costa Rica, hay un predominio de los fondos de la seguridad social. Haití tiene un alto componente de otras fuentes privadas, mientras que el gasto en seguros privados tiene un peso destacado en los Estados Unidos de América. En solo cinco países, Canadá, Costa
Rica, Cuba, Estados Unidos de América y Uruguay el gasto público sanitario está por encima de $6 \%$ del PIB (9).

Los gobiernos de las Américas destinan al sector salud, como promedio, $14 \%$ del gasto público como porcentaje del gasto total, en un rango de $7 \%$ a $23 \%$. El gasto de bolsillo es una barrera para el acceso y se considera la fuente de financiamiento más regresiva e ineficiente. El monto de este gasto tiene también una gran variabilidad en la Región, de 4,4\% en Cuba, seguido de Estados Unidos, Surinam, Canadá, Colombia y Uruguay, que se encuentran entre $10 \%$ y $20 \%$. El resto de los países de América Latina presentan una mayor variabilidad, a Venezuela le corresponde el mayor porcentaje por encima de $60 \%$ (9).

No existe un camino único para avanzar hacia la Salud Universal, porque en cada país existen factores contextuales que condicionan esa estrategia. Un sistema de salud es eficiente si logra conjugar una mejoría continua en sus indicadores de salud y un incremento del bienestar sin riesgos financieros para su población de modo que, para unos recursos dados, se optimicen los resultados en términos de indicadores sanitarios, con un acceso equitativo a los servicios de salud. Un sistema ideal de este tipo orienta sus políticas a producir lo que la sociedad necesita y espera en materia de salud y bienestar y en esa responsabilidad se ven implicados el conjunto del estado y la sociedad.

En Cuba, se obtuvieron muchos de los resultados de orden cuantitativo de la Salud Universal. Exhibe indicadores de salud que la sitúan en niveles comparables a los de muchos países desarrollados sin que haya brechas en relación con ninguno de los criterios normativos para la medición de las inequidades como el género, la educación, el ingreso, la ocupación, la localización urbana o rural y otros. Esta salud gratuita, equitativa y no excluyente ubica al país de manera favorable para el alcance de las metas de la Salud Universal $(10,11)$.

\section{El financiamiento de la salud pública cubana}

El financiamiento de la salud pública corre por cuenta del Estado cubano; los servicios de salud son estatales, mediante la red asistencial del SNS. Todos sus gastos se cubren a través del presupuesto del Estado asignado al sector salud (12).

Un elemento importante en los sistemas sanitarios es la financiación de la 
atención de la salud, que se centra en la movilización, la acumulación y la asignación de recursos para cubrir las necesidades de salud de la población (13). El sistema de financiación cubano está diseñado para proporcionar a toda la población el acceso a servicios de calidad y garantizar que el uso de estos servicios no exponga al usuario a dificultades financieras. Además, tiene definida la implementación de políticas y acciones con un enfoque multisectorial y transdisciplinario, para abordar los determinantes sociales de la salud y fomentar el bienestar de la sociedad, lo cual está en consonancia con lo planteado por OPS respecto al financiamiento de los sistemas y servicios de salud.

El SNS constituye un modelo a seguir según la perspectiva de investigadores y analistas en salud pública (14). Se basa en la medicina preventiva y brinda servicios no solo dentro del contexto nacional, sino también a países del tercer mundo. También tiene peculiaridades en la estrategia de formación de recursos humanos para la salud, pues las universidades de ciencias médicas pertenecen al SNS con el asesoramiento de los Ministerios de Educación y Educación Superior en el orden pedagógico y metodológico. Toma en cuenta el perfil epidemiológico del país en lugar de atenerse a condicionamientos de mercado y bajo condiciones solidarias, se abre a jóvenes del mundo entero que en otros contextos no pudieran acceder a estudios de este tipo.

Se expone información acerca del financiamiento de la salud pública cubana, mediante el análisis de las series temporales del gasto en salud total y expresada como porcentaje del PIB entre 1996 y 2015 (figura 1).

La Ley del Presupuesto del Estado, así como el presupuesto aprobado para 2017 para el sector de la salud, permiten afirmar que la tendencia al crecimiento de los gastos ha continuado hasta el presente. El presupuesto del Estado cubano es el plan fundamental que norma la distribución de una parte sustancial del ingreso nacional destinado al fomento de la economía, al incremento del bienestar material y cultural de la sociedad, a la defensa nacional y al funcionamiento de los órganos y organismos del Estado. El análisis de su ejecución, en especial en lo que se refiere a los gastos, es el principal instrumento de que se dispone para identificar la prioridad fiscal en la utilización de los recursos financieros (15).

El gasto en salud pública y asistencia social, en el período presentado, estuvo condicionado por varios factores. Aun cuando la relación no es determinista, su influencia fue considerable. Dos de estos factores fueron especialmente influyentes: la crisis económica de inicios de la década de los 90, consecuencia de la desaparición del campo socialista en

FIGURA 1. Gasto en salud y asistencia social, 1996-2015

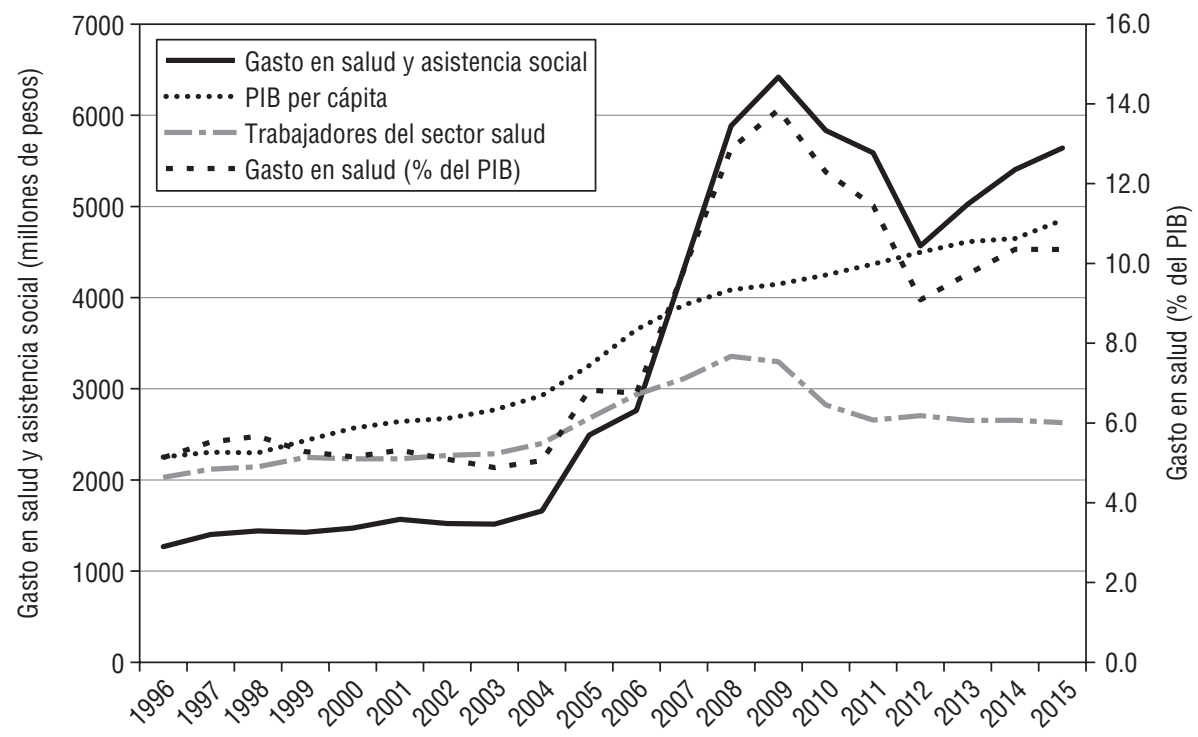

a Tasa de cambio: 1 peso cubano = 1 dólar estadounidense. Precios constantes 1997. Deflactor PIB. ONEI. Fuente: elaboración de los autores a partir de las fuentes de la Oficina Nacional de Estadísticas e Información (ONEI) y el Ministerio de Salud Pública (MINSAP).

Europa (16) y un fuerte proceso inversionista en infraestructura y tecnología médica, entre 2004 y 2009, dirigido a atenuarlos efectos negativos del decenio anterior (17). A partir de 2010, se produce un decrecimiento a expensas de la fuerza de trabajo hasta 2012, para volver a crecer en los años más recientes.

El gasto público expresado como porcentaje del PIB es uno de los indicadores más utilizados como expresión de la importancia que los gobiernos conceden a la salud como parte de sus políticas públicas. En juicios comparativos con otros países del área, los valores del gasto en salud como porcentaje del PIB son elevados (18). Los países con "desarrollo humano muy alto" y los que pertenecen a la Organización para la Cooperación Económica y el Desarrollo (OECD) exhiben valores por encima del $7,0 \%$, mientras los países con "desarrollo humano medio" y "desarrollo humano bajo", los "menos desarrollados" y los del sur de Asia presentan valores por debajo del 2,0\%. El valor global es $6,0 \%$, que coincide con la magnitud observada en documentos relativos a la Salud Universal y se señala como umbral a alcanzar (cuadro 1) (18).

$\mathrm{Al}$ analizar el comportamiento del gasto en salud y asistencia social como porcentaje del PIB se constata que, entre 1996 y 2004, este indicador toma valores relativamente bajos, aunque cercanos al $6 \%$ y luego aumenta, de forma sostenida hasta el 13,8\% en el año 2009, decrece a partir de ese año hasta 9,1\% (producto de ajustes al presupuesto salarial) y se recupera a 10,4\% en el 2014 debido al incremento salarial al personal de salud (7). Se incluye también el componente gratuito de ayuda solidaria que presta Cuba a muchos países del mundo de manera permanente o bajo condiciones no previsibles de ayuda humanitaria. Esto se justifica por la responsabilidad del Estado cubano en priorizar la salud de la población (figura 1).

En Cuba, el salario es un importante componente del gasto en salud, debido a la gran cantidad de trabajadores del sistema. El número de trabajadores en el sector de la salud observan una tendencia creciente en correspondencia con los gastos de salud hasta el año 2010. A partir de ese año, la nómina de trabajadores del sistema se redujo en más de 150000 plazas, producto del proceso de las transformaciones ejecutadas en el sector, la mayoría de las plazas sujetas al ajuste fueron administrativas y de 
CUADRO 1. Gasto público en salud, 2014

\begin{tabular}{lc}
\hline Clasificaciones & $\begin{array}{c}\text { Gasto público en salud } \\
\text { (\% del PIB) }\end{array}$ \\
\hline Grupos de desarrollo humano & 7,5 \\
Desarrollo humano muy alto & 3,4 \\
Desarrollo humano alto & 1,8 \\
Desarrollo humano medio & 1,7 \\
Desarrollo humano bajo & 3,0 \\
Países en desarrollo & 3,0 \\
Regiones & 3,0 \\
Estados árabes & 3,7 \\
Este de Asia y Pacífico & 3,6 \\
Europa y Asia central & 1,6 \\
América Latina y el Caribe & 2,4 \\
Sur de Asia & 1,8 \\
África subsahariana & 5,3 \\
Países menos desarrollados & 7,7 \\
Estados en desarrollo en islas pequeñas & 10,4 \\
Organización para la cooperación económica y el desarrollo (OECD) & 6,0 \\
Cuba & 3 \\
Mundo & \\
\hline
\end{tabular}

PIB, producto interno bruto.

${ }^{a}$ Gastos en salud, corrientes y de capital, de los presupuestos del gobierno (central y local), préstamos y subsidios externos (incluidas donaciones de agencias internacionales y organizaciones no gubernamentales) y fondos de seguro social (u obligatorio) de salud, expresados como porcentaje del PIB.

Fuente: Elaboración de los autores a partir de UNDP. Human development report, 2016. Table 8. Health outcomes, p. 231 y la ONEI para el caso Cuba.

FIGURA2. Distribución porcentual de los gastos de la actividad presupuestada, según partidas seleccionadas, 2008-2015

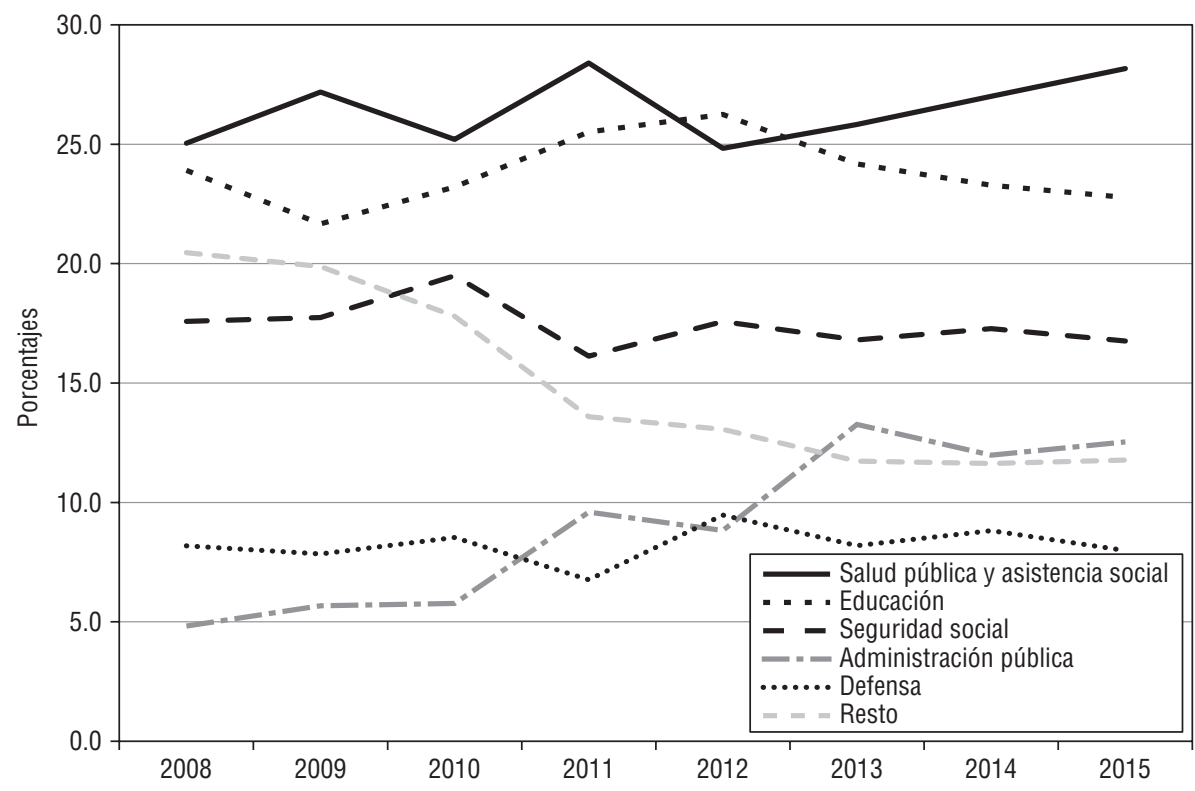

a Tasa de cambio: 1 peso cubano = 1 dólar estadounidense. Precios constantes 1997. Deflactor PIB. ONEI. Fuente: elaboración de los autores a partir de las fuentes de la Oficina Nacional de Estadísticas e Información (ONEI) y el Ministerio de Salud Pública (MINSAP).

trabajadores no vinculados directamente los procesos de la atención en salud (6). Ello permitió una recuperación de la ejecución presupuestaria y el gasto por habitante, relacionados con una política de incrementos salariales en el sistema de salud y con nuevas inversiones (3).

Otro aspecto a analizar es la distribución de los gastos de la actividad presupuestada ${ }^{5}$, en partidas del presupuesto seleccionadas que muestra la información oficial disponible acerca de la distribución de los gastos estatales según la actividad presupuestada, entre 2008 y 2015 (figura 2) (7).

La salud pública y la asistencia social han tenido una alta priorización fiscal, con porcentajes que, por lo general, sobrepasan $25 \%$ del total de gasto de la actividad presupuestada; se conoce que para el 2017 este valor llega a $28 \%$, solo es comparable con la educación, otro importante objetivo de la política cubana cuya influencia sobre la salud pública está fuera de toda duda. Debe constatarse como positivo que las carteras de Defensa y Seguridad Social se hayan mantenido estables. Sin embargo, llama la atención que las áreas de ciencia e innovación tecnológica, que se incluye en la categoría "Resto", haya tenido una tendencia decreciente (7).

Otro elemento relacionado con el financiamiento es el gasto de bolsillo por concepto de salud. Sin embargo, el país no dispone de un sistema de cuentas nacionales de salud, ni tiene información de resultados de encuestas al hogar que permita tener una visión objetiva y fiable de este indicador.

La principal fuente de erogación familiar en Cuba se asocia con los procesos de envejecimiento poblacional y a los escasos recursos con que cuenta el país para atender a una población con alta expectativa de vida $y$, por consiguiente, con una mayor carga de las comorbilidades asociadas a los grupos de edad avanzada para los que la atención médica hospitalaria -aunque esté garantizada- no es el recurso que se necesita. Por lo general, la familia debe soportar una carga económica en este aspecto. Existen estudios en los que se señala que el gasto de bolsillo es un indicador sensible a la economía familiar de los cubanos y que, por lo tanto, debe considerarse en los análisis económicos del sector (19).

\section{La eficiencia y otros retos económicos de la salud pública en Cuba}

El derecho a la salud, la equidad y la solidaridad son principios y valores

5 Se refiere a las actividades de las unidades que reciben presupuesto del Estado. Incluyen actividades productivas, servicios empresariales, actividades inmobiliarias y de alquiler, administración pública, defensa, seguridad social, ciencia e innovación tecnológica, educación, salud pública y asistencia social, cultura y deportes, y otras actividades de servicios comunales, de asociaciones y personales. 
esenciales de la salud universal que Cuba hizo suyos mediante un ejercicio consecuente que desborda sus fronteras, pero que colocó al sistema nacional de salud en una permanente tensión para conciliar estos valores con la eficiencia. La OPS/OMS los reconoce como necesidad de cambio hacia un nuevo paradigma de protección social basado en los tres principios y valores antes mencionados (20).

En Cuba, estos principios y valores forman parte del sistema de salud y están explícitos en su carta magna y en su base jurídica, no se basa en una lógica contributiva y, por consiguiente, ha exigido una búsqueda constante del fortalecimiento y optimización del sistema público, preservando la no exclusión y promoviendo políticas intersectoriales alineadas con el principio de los determinantes sociales de la salud (21).

La eficiencia es un tema que tiene cada vez mayor presencia en las agendas de los encargados de la toma de decisiones en los sistemas sanitarios $(20,22)$. Se estima que entre $20 \%$ y $40 \%$ del gasto sanitario de los países se desaprovecha por la ineficiencia (10). Para entender mejor el significado de los porcentajes mencionados, basta con la estimación aproximada de que si, en 2015, 30\% de la asignación presupuestaria ejecutada en Cuba para Salud Pública y Asistencia Social se hubiera perdido por ineficiencia, ello habría representado, en números redondos, 3200 millones de pesos cubanos. Ningún país de la región o del mundo puede permitirse esas cifras.

Según el MINSAP, en 2015, 42,6\% del presupuesto en salud se asignó al nivel primario de atención, que resuelve la mayoría de los problemas de salud de la población (12). Cualquier acción que pueda solucionarse en este nivel y se derive a otro, impone un ingrediente de derroche e ineficiencia para el sistema sanitario. Priorizar la inversión en este nivel de atención, elevar el perfil epidemiológico, unido a la gratuidad de los servicios, la eliminación de barreras económicas, focalización y protección de grupos vulnerables o con mayores niveles de exposición al riesgo han sido una prioridad y se incluyen en las estrategias fundamentales para alcanzar la salud universal que garantizan un primer nivel de atención que no solo es fuerte y resolutivo sino que, además, es el eje de articulación de las redes integradas de servicios.
En la mayoría de los países de la Región de las Américas, una de las más importantes fuentes de ineficiencia es la existencia de sistemas de salud segmentados y fragmentados. En Cuba, la segmentación se eliminó poco después de 1959, cuando el Estado asumió la mayor carga presupuestaria del gasto en salud. La fragmentación desapareció cuando se decidió la integración del SNS en 1970 y, definitivamente, con la creación del modelo cubano de medicina familiar en 1984 (23).

Un signo de madurez del SNS es la capacidad de identificar los factores que afectan la eficiencia y de adoptar medidas para rectificarlos. Este objetivo se ha incorporado de modo explícito en los programas de salud (24). Una especial significación ha tenido el Programa por la eficiencia y uso racional de recursos (25) que, desde 2009, incluye entre sus propósitos la mejora de la calidad de los servicios a partir del uso inteligente de los recursos, el perfeccionamiento de las plantillas laborales de las instituciones de salud, el plan de estímulos para los trabajadores del sector, el uso racional de infraestructuras, el perfeccionamiento de sistemas de costos en unidades del SNS y el control del despilfarro y las ilegalidades (26).

Existe, además, un Programa Nacional de Medicamentos mediante el cual se controla el proceso desde la prescripción médica basada en fármacos aprobados en función de morbilidades específicas, hasta el nivel de su distribución. Entre sus normativas, se encuentra la formulación de un cuadro básico de medicamentos que se analiza y se ajusta en forma anual $(27,28)$.

A pesar de los logros alcanzados, no hay dudas que existen urgentes desafíos en el área económica para alcanzar mejores resultados:

- Integrar las estrategias de Salud Universal al proceso de desarrollo del SNS y alinearlas en correspondencia con el nuevo modelo económico de Cuba.

- Sistematizar el uso de la evaluación económica y de tecnologías sanitarias como un instrumento para la toma de decisiones.

- Fortalecer la intersectorialidad como capacidad de respuesta para enfrentar los problemas sanitarios, y como base de integración de los sectores de la economía y la sociedad con el sector de la salud.
- Fomentar el estudio sobre sistemas de pago como mecanismo de incentivo a la eficiencia, en particular el pago por resultados, no solo para los recursos humanos de salud sino también para el financiamiento de las instituciones de salud.

- Desarrollar un sistema de cuentas nacionales en salud que contribuya al perfeccionamiento de la planificación, la formulación y el monitoreo de nuevas estrategias sectoriales, la distribución de recursos y el perfeccionamiento de la contabilidad social.

Se espera que el análisis realizado, y en particular las experiencias expuestas, contribuya al intercambio con la comunidad científica sobre algunos temas cruciales, al ofrecer un panorama actual más claro de la realidad cubana; no solo en cuanto a conquistas y realizaciones, sino de barreras a superar, que se resumen en forma de lecciones aprendidas (cuadro 2).

\section{CONCLUSIONES}

La Salud Universal en Cuba se considera una de sus más valiosas realizaciones, aunque requiere del análisis más eficiente de fuentes fiables y disponibles apenas explotadas; la investigación y aplicación de resultados a la práctica sobre las fortalezas y debilidades del sistema en materia de economía de la salud. Es imprescindible además actualizar los recursos y modernizar las capacidades analíticas.

El trabajo expuso las acciones fundamentales en el orden económico en el contexto de la estrategia de Salud Universal, con énfasis en el financiamiento, eficiencia y desafíos de la salud pública cubana para mantener el derecho a la salud de sus ciudadanos, la equidad, cobertura y acceso en los servicios que se prestan, la eliminación de las barreras económicas, socioculturales y de género; así como la solidaridad con la población cubana y del mundo.

Para ello, profundiza en las lecciones aprendidas y enfrenta un conjunto de desafíos que permiten el monitoreo permanente de indicadores de la estrategia de Salud Universal en cuanto al fortalecimiento y desarrollo de instrumentos financieros, la optimización del financiamiento en salud, el desempeño resolutivo del primer nivel de atención y la 
CUADRO 2. Lecciones aprendidas según el análisis de sus dimensiones y acciones

\begin{tabular}{|c|c|c|}
\hline Dimensiones & Análisis & Acciones \\
\hline $\begin{array}{l}\text { Sistema de salud cubano y la } \\
\text { Salud Universal }\end{array}$ & $\begin{array}{l}\text { Se evidencia la necesidad de integrar las estrategias de Salud } \\
\text { Universal al proceso de desarrollo del SNS y alinearlas en } \\
\text { correspondencia con el nuevo modelo económico de Cuba } \\
\text { Se evidencia la importancia de fomentar la toma de decisiones con } \\
\text { perspectiva económica }\end{array}$ & $\begin{array}{l}\text { Se desarrolló un proceso de transformaciones y mejoras continuas } \\
\text { en el sector de la salud que tiene en cuenta las estrategias de Salud } \\
\text { Universal, en aras de incrementar la calidad de la atención, la } \\
\text { efectividad y la eficiencia } \\
\text { Se implementó un programa nacional por el uso racional y eficiente } \\
\text { de los recursos } \\
\text { Se produjo un proceso de capacitación y fortalecimiento de las } \\
\text { competencias de los directivos y funcionarios del SNS }\end{array}$ \\
\hline Financiamiento del SNS & $\begin{array}{l}\text { Se constata que disponer de un adecuado soporte financiero para la } \\
\text { gestión del SNS requiere del fortalecimiento de los mecanismos de } \\
\text { control del presupuesto }\end{array}$ & $\begin{array}{l}\text { Se fortalecieron los mecanismos para el control del presupuesto y } \\
\text { otros procesos económicos en todos los planes y acciones del } \\
\text { SNS } \\
\text { Se integraron nuevas fuentes de financiamiento en aras de la } \\
\text { sostenibilidad del SNS }\end{array}$ \\
\hline & $\begin{array}{l}\text { Se aprecia que la escasez de recursos y su dificultad de obtención } \\
\text { pueden convertirse en generadores para encontrar reservas de } \\
\text { eficiencia }\end{array}$ & $\begin{array}{l}\text { Se incluyeron en el programa nacional el uso racional y eficiente de } \\
\text { los recursos nuevos métodos, mecanismos y formas de } \\
\text { racionalización para una mejora continua de los procesos }\end{array}$ \\
\hline Eficiencia en el SNS & $\begin{array}{l}\text { Se visualiza la necesidad de fomentar la eficiencia a partir de trabajar } \\
\text { en áreas programáticas específicas en función del uso inteligente de } \\
\text { los recursos. } \\
\text { El enfrentamiento a los problemas sanitarios es responsabilidad de } \\
\text { toda la sociedad. }\end{array}$ & $\begin{array}{l}\text { Se identificaron y fortalecieron áreas programáticas que tributan al } \\
\text { perfeccionamiento y uso inteligente de los recursos, se prioriza el } \\
\text { programa de medicamentos por su alta sensibilidad en el SNS } \\
\text { Se perfeccionaron estrategias de trabajo intersectorial para el } \\
\text { enfrentamiento a los problemas sanitarios }\end{array}$ \\
\hline
\end{tabular}

SNS, sistema nacional de salud.

alineación del modelo económico cubano en cuanto a mecanismos de pago y presupuesto estatal, entre otros.

Agradecimientos. Los autores agradecen a Cristián Morales Fuhrimann, Justo th coverage and universal access [Editorial]. Bull World Health Organ. 2013. Disponible en: http://www.who.int/bulletin/volumes/91/8/13125450.pdf Acceso el 19 de abril de 2017.

2. Organización Panamericana de la Salud (OPS). CD53.R14: estrategia para el acceso universal a la salud y la cobertura universal de salud. Washington D.C.: OPS; 2014. Disponible en: http://iris.paho.org/xmlui/ handle/123456789/7652?show=full Acceso el 18 de abril de 2017.

3. Ministerio de Salud Pública de Cuba (MINSAP). Dirección de Registros Médicos y Estadísticas de Salud. Anuario Estadístico de Salud, 2016. La Habana: MINSAP; 2017.

4. Ministerio de Salud Pública de Cuba (MINSAP). Informe de Balance Anual del MINSAP, 2017.

5. Rodríguez JL. La economía cubana 2016-2017. Valoración preliminar (II). Disponible en: http://www.cubadebate. cu/opinion/2017/01/18/laeconomiacubana20162017valoracionpreliminarii/\#.WNOjsuKDeUk Acceso el 13 de marzo de 2016.

6. Morales Ojeda R, Más Bermejo P, CastellFlorit Serrate P, Arocha Mariño C, Valdivia Onega NC, Druyet Castillo D, et al. Transformaciones en el sistema de salud en Cuba y estrategias actuales para su consolidación y sostenibilidad. Rev Panam Salud Publica. 2018;42:e25. doi: 10.26633/ RPSP.2018.25
Manuel Cárdenas Rodriguez y Jorge Bacallao Gallestey por sus valiosas recomendaciones.

Conflicto de intereses. Ninguno declarado por los autores.

\section{REFERENCIAS}

7. Oficina Nacional de Estadística e Información (ONEI). Anuario Estadístico de Cuba 2015. La Habana: ONEI; 2016. Disponible en: http://www.one.cu/aec2015.htm Acceso el 18 de abril de 2017.

8. Oficina Nacional de Estadística e Información (ONEI). Cuba: principales indicadores económicos. En: Oficina Nacional de Estadística e Información. Panorama económico y social. Cuba 2015. La Habana: ONEI; 2016. Disponible en: http://www.one.cu/ publicaciones / 08informacion/panorama2015/Panorama2015.pdf Acceso el 18 de abril de 2017.

9. Global Health Expenditure Database [base de datos en internet], World Health Organization (WHO). Geneve: WHO; 2014. Disponible en: http://apps.who.int/nha/ database/ViewData/Indicators/es Acceso el 3 de marzo de 2017.

10. Pinzón Flórez CE, Chapman E, Panisset U, Arredondo A, Fitzgerald J, Reveiz L. Disponibilidad de indicadores para el seguimiento del alcance de la "Salud Universal" en América Latina y el Caribe. Rev Panam Salud Publica. 2016;39(6):330-40. Disponible en: http://iris.paho.org/xmlui/handle/123456789/28548 Acceso el 18 de abril de 2017.

11. World Health Organization (WHO). Global Health Observatory data repository. Health Expenditure ratios, by country 1995-2014, Cuba. Disponible en: http:// apps.who.int/gho/data/view.main.HEALLTHEXPRATIOCUB Acceso el 20 de abril de 2017.
Declaración. Las opiniones expresadas en este manuscrito son responsabilidad de los autores y no reflejan necesariamente los criterios ni las políticas de la RPSP/PAJPH y/o de la OPS.
12. Ministerio de Salud Pública de Cuba (MIN$\mathrm{SAP}$ ). Área de Economía. Información Económica. La Habana: MINSAP; 2016.

13. Organización Panamericana de la Salud/ Organización Mundial de la Salud (OPS/ OMS). Financiamiento de los sistemas de salud. [Internet]. Washington D.C.: OPS/ OMS; 2017. Disponible en: http://www2. paho.org/hq/index.php?option=com_cont ent\&view $=$ article\&id $=2973 \% 3$ Ahealthfinancing\&catid $=524 \% 3$ Ahealtheconomicsfinancing\&Itemid $=2080 \&$ lang $=$ es Acceso el 3 de marzo de 2017.

14. Lamrani S. El sistema de salud cubano, un modelo según la OMS. Artículo de opinión. Disponible en: http:/ / www.tercerainformacion.es/antigua/spip.php?article71890 Acceso el 20 de setiembre de 2017.

15. Gaceta oficial de la República de Cuba. Ley No. 122 del Presupuesto del Estado para el año 2017. Disponible en: http:// www.one.cu/publicaciones/08informacion/Presupuesto $\% 202017 /$ Ley $\% 20$ No. \%20122\%20Presupuesto\%202017.pdf Acceso el 13 de marzo de 2017.

16. Rodríguez JL. La desaparición de la URSS 25 años después: algunas reflexiones (I) Disponible en: http://www.cubadebate. cu/opinion/2016/05/07/la-desaparicion-de-la-urss-25-anos-despues-algunas-reflexiones-i/\#.WZ2rpfjyjcs Acceso el 13 de abril de 2017.

17. González Rodríguez A. Inversiones, reparación y mantenimiento en instituciones de salud: otro logro de 2016. Disponible en: http://www.radiococo.icrt.cu/noticias/ 
salud/inversiones-reparacion-mantenimiento-instituciones-salud-logro-2016/ Acceso el 18 de abril de 2017.

18. Programa de las Naciones Unidas para el Desarrollo (PNUD). Human Development Report Expenditure on health, total (\% of GDP). Disponible en: http://hdr.undp. org/es/content/expenditurehealthtotalgdp Acceso el 13 de marzo de 2017.

19. Fleitas Ruiz R, Ávila Vargas N, Solares Pérez L, Hernández Arencibia R, Chaviano Balmaseda A. Los gastos de salud en familias cubanas. Estudios de casos. En: Fleitas Ruiz R, compiladora. Investigación socioantropológica en salud. Enfoque de género. La Habana: Centro de Estudios Demográficos; 2015:111-22.

20. World Health Organization (WHO). Health in All Policies (HiAP): framework for country action. Geneve: WHO; 2014.

21. Castell Florit P. Sostenibilidad de la intersectorialidad en Cuba. La Habana: Editorial de Ciencias Médicas; 2009.

22. Cid C, Báscolo E, Morales C. La eficiencia en la agenda de la estrategia de acceso y cobertura universales en salud en las Américas. Salud Publica Mex. 2016;58(5):496503.
Disponible en: http://saludpublica.mx/ index.php/spm/article/view/8182/10824 Acceso el 28 de marzo de 2017.

23. Presno Labrador C. El médico de familia en Cuba. Rev Cubana Med Gen Integr. 2006;22(1): [aprox. 7 p.]. Disponible en: http:/ / scielo.sld.cu/scielo.php?script=sci arttext\&pid=S086421252006000100015\&ln$\mathrm{g}=\mathrm{es}$ Acceso el 18 de abril de 2017.

24. Ministerio de Salud Pública de Cuba (MINSAP). Transformaciones necesarias en el Sistema de Salud Pública. La Habana: MINSAP; 2010. Disponible en: http:// files.sld.cu/editorhome/files/2010/11/ transformaciones-necesarias-salud-publica.pdf Acceso el 6 de octubre de 2017.

25. Ministerio de Salud Pública de Cuba (MINSAP). Programa por la eficiencia y el uso racional de los recursos. La Habana: MINSAP; 2009.

26. Contraloría General de la República de Cuba. Sistema de control interno. La Habana, 2012. Disponible en: http://www. contraloria.cu/documentos/folletoSistemaCl.pdf Acceso el 25 de marzo de 2017.
27. Ministerio de Salud Pública de Cuba (MINSAP). Programa Nacional de Medicamentos. La Habana: Editorial Ciencias Médicas; 2014. Disponible en: http://instituciones. sld.cu/hospmiguelenriquez / files/2016/05/Programanacionalde$\mathrm{m}$ e d i c a m e $\mathrm{n}$ t o s V I v e r s i \% C3\%B3n.pdf Acceso el 28 de marzo de 2017.

28. Lara Bastanzuri MC, Calvo Barbado D, Broche Villareal L, Perdomo Delgado J. Cuadro Básico de Medicamentos 2016. Boletín Información terapéutica para la APS, 2016. Disponible en: http://files.sld.cu/ cdfc/files/2016/03/boletinapsno37marzo2016web.pdf Acceso el 28 de marzo de 2017

Manuscrito recibido el 28 de abril de 2017. Aceptado para publicación, tras revisión, el 12 de diciembre de 2017. siderations and economic actions on financing, efficiency and other economic challenges of public health in Cuba. Official bibliographic sources of the Cuban state were revised up to 2015. The analyses of time series were adjusted to constant prices of 1997

\section{Economic considerations on Cuban public health and its relationship with universal health} and the official exchange rate of 1 Cuban peso $=1$ US dollar. The Cuban National Health System has a solid infrastructure, consisting of a wide network of health facilities that guarantee full coverage, access and equity in the services provided to the population through qualified human resources. An economic analysis was carried out under the conceptual framework of the universal health strategy, with an emphasis in financing, efficiency and challenges of the Cuban public health system to maintain the right to health of its citizens, equity, coverage and access to the services provided, including the elimination of economic, sociocultural and gender barriers, as well as solidarity with the Cuban population and the world. It was concluded that in Cuba, universal health is considered one of its most valuable achievements, although it is needed a most efficient analysis of reliable and available sources, research and application of results to practice on the strengths and weaknesses of the system regarding health economics, updating and a better use of resources and technologies, as well as deepening the lessons learned and facing remaining challenges.

Keywords Health care financing; efficiency; health economics; Cuba. 
RESUMO Este artigo apresenta, no contexto da estratégia da saúde universal, algumas considerações gerais e ações econômicas sobre financiamento, eficiência e outros desafios econômicos da saúde pública em Cuba. As fontes bibliográficas oficiais do estado

Considerações econômicas sobre a saúde pública cubana e sua relação com a saúde universal cubano foram revisadas até 2015. As análises das séries temporais foram ajustadas aos preços constantes de 1997 e à taxa oficial de câmbio 1 peso cubano $=1$ dólar norteamericano. O Sistema Nacional de Saúde cubano possui uma sólida infra-estrutura, constituída por uma ampla rede de instalações de saúde que garantem cobertura total, acesso e equidade nos serviços da população com recursos humanos qualificados. Uma análise econômica foi realizada no marco conceitual da estratégia de saúde universal que enfatizou o financiamento, eficiência e desafios da saúde pública cubana para manter o direito à saúde de seus cidadãos, equidade, cobertura e acesso no serviços prestados, eliminação de barreiras econômicas, socioculturais e de gênero; bem como a solidariedade com a população cubana e o mundo. Concluiu-se que, em Cuba, a saúde universal é considerada uma das suas conquistas mais valiosas, embora requer a análise mais eficiente de fontes confiáveis e disponíveis, pesquisa e aplicação de resultados para a prática dos pontos fortes e fracos do sistema em termos de economia da saúde, atualização e uso de recursos e tecnologias, além de aprofundar as lições aprendidas e enfrentando um conjunto de desafios.

Palavras-chave Financiamento da assistência à saúde; eficiência; economía da saúde; Cuba. 